\title{
Health Care Systems and Transfer of Blood Transfusion Service to Sudan
}

\author{
Ihab El-Hemaidi \\ Queen Elizabeth Hospital London, UK
}

\begin{abstract}
A successful healthcare system is a vital element in a successful healthcare plan. The importance of a successful healthcare system lies in its ability in attracting and retaining talented healthcare professional. In this paper I argue the case for developing primary healthcare and tertiary healthcare in this first phase of development of the healthcare system and postponing the development of secondary care to the next phase. Blood transfusion service is an essential basic component of any healthcare system. In this document I argue the case of changing the current fragmented blood transfusion service to a more centralised service. This change will improve the blood transfusion safety, supplies and funding of the system.
\end{abstract}

\section{Introduction and Objectives}

This document addresses 2 major issues that face developing healthcare systems:

1) The role that Diaspora could play in helping to shape the healthcare system in the countries of their origin in a positive way. This role could be targeted to improve governance, fairness in distribution of services, efficacy in delivery of healthcare service, accountability, transparency and its cost-effectiveness.

2) The second component is on the optimal way of delivering a safe effective blood transfusion service in Sudan within an effective functional healthcare system.

\section{Discussion}

An accepted and functioning Healthcare system is integral part of any successful healthcare plan in any country. Success of a healthcare system is vital and is dependent on many factors which include the acceptance and the support for the system:

- $\quad$ By the general public,

- $\quad$ By the Healthcare workers and

- $\quad$ By the different political parties of the country.

The importance of an accepted, supported and efficient healthcare system can't be stressed enough. Its success is vital in attracting and retaining the creative and talented healthcare professionals whose contributions are essential in the success of any healthcare system. The role of the healthcare professionals is to lead a service that delivers to its end users the required services. A successful healthcare system should also be able to be responsive to the needs of individuals and communities. The positive role that Diaspora could play is to share their experiences through the appropriate networks with their counterparts in the Sudan. These networks would enrich the ideas of both groups and lead to a better understanding of problematic issues and this would in turn lead to devising effective solutions to difficult problems. 
My personal proposal for the healthcare system in Sudan at this stage of its development is to focus on developing an effective primary healthcare system. Primary healthcare system is the backbone of any healthcare system it should be built in a way that it is able to adapt to local needs of each Sudanese region while at the same time being able to address the major health challenges that face Sudan as a nation. Successful primary healthcare systems should also be able to gather statistical data of the nation's health. This would enable policy makers in directing resources according to the nations needs.

I would also argue that developing a capable tertiary healthcare system is important at this stage. An effective tertiary healthcare system would stop the haemorrhage of hardly earned currencies to other countries. This service in time could act a as a magnet towards earning the country much needed hard currency from the neighbouring countries. Tertiary healthcare system would have the ability of becoming a beacon of knowledge from which the light of knowledge could be dispersed to various parts of the country. Improving and developing secondary care could be postponed to a later phase in the healthcare development cycle after defining the exact needs from this sector.

Blood transfusion is one of the essential basic services in any health care system. Its importance lies in its supportive function towards other medical specialities. Its importance for all different medical specialities Obstetrical and Gynae, Surgery, Paediatrics and general medicine can't be emphasised enough. From a public health prospective it is of utmost importance to establish an effective, safe, cost-effective service supported by the tax payer.

Currently blood transfusion service is fragmented and each hospital has its own system of blood donor selection, exclusion and testing. The current system partially fulfils the blood transfusion requirements in larger hospitals in larger cities however it is inadequate when there are shortages in blood supplies. It is also inadequate and inefficient system when implemented in smaller hospitals and when it is required to produce information on levels of blood supplies in each region of the country.

I would propose a more centralised blood transfusion system. In this system Sudan is divided into 8 regions each region with 5 million inhabitants. Each region would have a central facility that is responsible for blood donor recruitment, selection, testing, venesecting, safe storage and issuing of blood to hospitals in its region. It would be very informative if the regions are connected and blood supplies could be shared between different regions in times of shortages. It would be advisable to start this massive project by building a pilot centre in Khartoum and to use this centre as learning model from which lessons could be learnt in the future when the project is rolled over to other regions of the Sudan.

A more centralised system that benefits from the economies of scale and the ability to automate would offer major advantage in terms of its ability to provide a cost-effective, safer blood supply to the whole country. In a centralised system it would be much easier to implement guideline and train staff. However a centralised system would need a major investment into infrastructure and training of staff. This is one of the very few areas in medicine whereby the capital investment needed could potentially be returned back within a short period of time. The return in capital could be achieved through selling of the unused plasma in the international markets. Inspecting the table below (Annual SHOT Report, 2007) ${ }^{1}$ it is evident that almost yearly in the

Serious Hazards of Transfusion (SHOT) is a professionally-led haemovigilance scheme which is based at the Manchester Blood Transfusion Centre and is affiliated to the Royal College of Pathologists. 
Yearly summary of issues by the four UK Board Services 1999-2007

\begin{tabular}{|l|c|c|c|c|}
\hline \multicolumn{1}{|c|}{ Year } & Red blood cells & Platelets & FFP & Cryoprecipitate \\
\hline $1999 / 2000$ & $2,737,572$ & 249,622 & 365,547 & 94,114 \\
\hline $2000 / 2001$ & $2,706,307$ & 250,259 & 374,760 & 95,456 \\
\hline $2001 / 2002$ & $2,679,925$ & 251,451 & 385,236 & 88,253 \\
\hline $2002 / 2003$ & $2,678,098$ & 251,741 & 377,381 & 92,768 \\
\hline $2003 / 2004$ & $2,607,410$ & 264,539 & 372,855 & 95,417 \\
\hline $2004 / 2005$ & $2,428,934$ & 258,528 & 313,019 & 102,719 \\
\hline $2005 / 2006$ & $2,316,152$ & 259,654 & 320,852 & 106,139 \\
\hline $2006 / 2007$ & $2,235,638$ & 255,747 & 306,444 & 116,672 \\
\hline
\end{tabular}

Source: SHOT Annual Report 2007

UK only 1 out of 7 units of plasma taken is used. Thus in a centralised service when one unit of blood is donated and then it is split into its components red cells, plasma and platelets there will be an excess of about 6 out of 7 plasma bags that is not used (currently in Sudan whole blood is given to patients which means that 6 out of 7 individuals are given plasma unnecessarily). There is an international market for properly collected plasma and the sale of unused plasma would pay in time for the capital spent at the outset.

Even in the absence of the capital investment needed to establish a centralised service there would be some health services e.g. National Health Service (NHS) or private companies that would be willing to invest into the infrastructure in return for free plasma supplies for a limited period of time. In the centralised blood transfusion service that I propose blood donations, testing and issuing of the blood components would be the responsibility of a central service. While administration and issuing of blood components would be the responsibility of hospital blood banks. In a centralised system safety would be enshrined into its guidelines and should be ensured by the issuing service. These safe guards would benefit the public at large and the whole of the healthcare system.

\section{Conclusion}

In order for such a system to be successful this would need the presence of the political will in the form of the consent of the department of health and department of finance. On my part I am willing to give necessary advice needed for the procurement of the necessary equipment, the writing of the necessary guidelines, and the training of staff. I am also willing to help in the negotiations with NHS or any private company in establishing the first phase of this massive project.

\section{Reference}

Serious Hazards of Transfusion (SHOT) (2008) Annual SHOT Report 2007 Available online at: http://www.shotuk.org/SHOT\%20Report\%202007.pdf, Published 7th July, 2008, ISBN 978-0-9558648-0-3. 